\title{
Reduced incidence of hemophagocytic lymphohistiocytosis in Japan during the COVID-19 pandemic
}

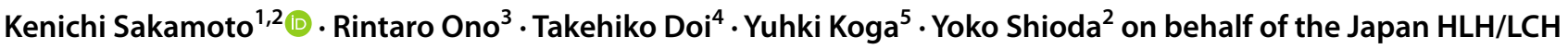 \\ consortium
}

Received: 24 November 2021 / Revised: 28 January 2022 / Accepted: 31 January 2022 / Published online: 3 February 2022

(c) Japanese Society of Hematology 2022

Hemophagocytic lymphohistiocytosis (HLH) has two distinct forms: primary HLH, which is a genetic disorder, and secondary HLH, which is due to infection, malignancy, autoimmune disorder, or stem cell transplantation. Ishii et al. [1] reported that $68.8 \%$ of patients diagnosed with HLH in Japan were under 30 years of age. The most frequent cause of HLH was Epstein-Barr virus (EBV). The sum of EBVassociated HLH (EBV-HLH) and other infection-associated HLH accounts for $53.1 \%$ of the total incidence of HLH in Japan. Development of primary HLH may also be triggered by infection. Thus, infectious etiologies are the main cause or trigger of HLH, especially in young children and adolescents.

In December 2019, the coronavirus disease 2019 (COVID-19) pandemic hit Japan [2]. Precautionary measures including proper handwashing, wearing of facemasks, and closure of schools were implemented to control the spread. The National Institute of Infectious Disease of Japan revealed that a majority of common infectious diseases (influenza, hand-foot and mouth disease, herpangina, infectious gastroenteritis, and respiratory syncytial virus infection) also markedly decreased in 2020 (during the COVID-19 pandemic).

Kenichi Sakamoto

sken1@belle.shiga-med.ac.jp

1 Department of Pediatrics, Shiga University of Medical Science, Seta Tsukinowa-Cho, Otsu, Shiga 520-2192, Japan

2 Department of Pediatrics, Children's Cancer Center, National Center for Child Health and Development, Tokyo, Japan

3 Department of Pediatrics, St. Luke's International Hospital, Tokyo, Japan

4 Department of Pediatrics, Hiroshima University Institute of Biomedical and Health Sciences, Hiroshima, Japan

5 Department of Pediatrics, Graduate School of Medical Sciences, Kyushu University, Fukuoka, Japan
We hypothesized that these changes in lifestyle and infectious disease incidence during the COVID-19 pandemic may have affected the incidence of HLH. Therefore, we assessed the incidence of six hematological diseases, namely HLH, infectious mononucleosis (IM), acute immune thrombocytopenia (aITP), Langerhans cell histiocytosis (LCH), acute lymphoblastic leukemia (ALL), and mature B cell lymphoma (B-NHL), before and during the COVID-19 pandemic (2018 to 2020). We analyzed the public dataset from the Blood Disease Registration maintained by the Japanese Society of Hematology (JSH). This dataset aggregates data from the JSH, Japanese Society of Pediatric Hematology and Oncology (JSPHO), and National Hospital Organization. From 2016 to 2019, the mean number of patients registered was $42,406 \pm 5,046$. In 2020 , this figure was 46,471 . To analyze changes in annual incidence, we calculated the mean and standard deviation for the 2016-2019 data, and compared this to the annual incidence of each disease in 2020.

The analyzed data are summarized in Table 1 . The total incidence of HLH decreased to $73.7 \%$, compared with the 2016-2019 mean incidence of HLH (152 vs. 206.3 \pm 10.2 ). Furthermore, HLH incidence in 2020 decreased by $77.5 \%$ in those less than 20 years old, and $77.9 \%$ in those over 20 years old. Similarly, the incidence of IM and aITP decreased by $91.5 \%$ and $78.4 \%$ in 2020 , compared to the respective mean incidences of IM and aITP from 2016 to 2019. The decrease in the number of IM and aITP patients was larger among those less than 20 years of age, compared to those 20 years of age or older (IM: $84.8 \%$ vs. $96.6 \%$, and aITP: $52.6 \%$ vs. $106.5 \%)$. On the other hand, the incidences of LCH, ALL, and B-NHL did not decrease in 2020 (LCH, 109.9\%; ALL, 102.9\%; and B-NHL, 111.9\%).

Our data revealed that the incidence of HLH decreased during the COVID-19 pandemic. We observed a similar trend in patients with IM and aITP. Various infectious diseases have dramatically decreased during the COVID-19 pandemic. In Japan, the most frequent cause of HLH in 
Table1 The incidence of hematological disease before and during COVID-19 pandemic

\begin{tabular}{|c|c|c|c|c|c|c|c|}
\hline Disease & $2016(N)$ & $2017(N)$ & $2018(N)$ & $2019(\mathrm{~N})$ & $\begin{array}{l}2016-2019(N, \\
\text { Mean } \pm \text { SD) }\end{array}$ & $2020(N)$ & $\begin{array}{l}\text { Rate of } \\
\text { change } \\
(\%)\end{array}$ \\
\hline \multicolumn{8}{|l|}{ Total } \\
\hline HLH & 219 & 209 & 195 & 202 & $206.3 \pm 10.2$ & 152 & -26.3 \\
\hline IM & 289 & 248 & 324 & 415 & $319.0 \pm 71.1$ & 292 & -8.5 \\
\hline aITP & 647 & 520 & 448 & 497 & $528.0 \pm 84.8$ & 414 & -21.6 \\
\hline $\mathrm{LCH}$ & 94 & 89 & 102 & 90 & $93.8 \pm 5.9$ & 103 & +9.9 \\
\hline ALL & 865 & 1152 & 1056 & 1083 & $1039 \pm 123$ & 1069 & +2.9 \\
\hline B-NHL & 15,472 & 17,005 & 19,239 & 20,474 & $18,047 \pm 2238$ & 20,198 & +11.9 \\
\hline \multicolumn{8}{|c|}{ Under 20 years } \\
\hline HLH & 97 & 79 & 65 & 75 & $74.8 \pm 13.3$ & 58 & -22.5 \\
\hline IM & 68 & 83 & 109 & 122 & $92.0 \pm 20.2$ & 78 & -15.2 \\
\hline aITP & 364 & 311 & 174 & 181 & $230.2 \pm 91.6$ & 121 & -47.4 \\
\hline $\mathrm{LCH}$ & 82 & 78 & 88 & 77 & $79.8 \pm 4.8$ & 74 & -7.3 \\
\hline ALL & 473 & 512 & 445 & 446 & $459.2 \pm 31.3$ & 420 & -8.5 \\
\hline B-NHL & 125 & 105 & 96 & 88 & $101.2 \pm 13.2$ & 92 & -9.1 \\
\hline \multicolumn{8}{|c|}{ Over 20 years } \\
\hline HLH & 122 & 130 & 130 & 127 & $120.6 \pm 13.6$ & 94 & -22.1 \\
\hline $\mathrm{IM}$ & 221 & 165 & 215 & 293 & $221.6 \pm 41.0$ & 214 & -3.4 \\
\hline aITP & 283 & 209 & 274 & 316 & $275.0 \pm 35.8$ & 293 & +6.5 \\
\hline $\mathrm{LCH}$ & 12 & 11 & 14 & 13 & $15.8 \pm 6.7$ & 29 & +83.5 \\
\hline ALL & 392 & 640 & 611 & 637 & $585.5 \pm 97.7$ & 649 & +10.8 \\
\hline B-NHL & 15,347 & 16,900 & 19,143 & 20,386 & $18,376 \pm 1948$ & 20,106 & +9.4 \\
\hline
\end{tabular}

$H L H$ hemophagocytic lymphohistiocytosis, IM infectious mononucleosis, aITP acute immune thrombocytopenia, $L C H$ Langerhans cell histiocytosis, $A L L$ acute lymphoblastic leukemia, $B$ - $N H L$ mature B cell lymphoma children and adolescents and young adults was infection, while malignancy (lymphoma)-associated HLH was the main cause of HLH in adults [1]. Thus, the annual incidence of HLH during the COVID-19 pandemic decreased due to a decrease in infectious triggers. EBV, which is the most frequent cause of HLH in Japan, is transmitted through saliva. Similar to EBV infection, the majority of these infections spread through contact and respiratory aerosol transmission. Lifestyle changes implemented during the COVID-19 pandemic, specifically social distancing, wearing of facemasks, alcohol disinfection, and school closure, effectively reduced contact and droplet transmission. The fact that incidence of IM and aITP also decreased during the COVID-19 pandemic decreased, especially in those younger than 20 years old, supports this hypothesis. Both IM and aITP are triggered by infection, and the majority of the cases occur in those less than 20 years old. In contrast, the incidence of LCH, ALL, and B-NHL, which are not triggered by infection, did not change during the COVID-19 pandemic.

This limitation of this report is as follows. First, the analyzed data from the Blood Disease Registration maintained by JSH were total numbers of HLH (both primary and secondary). Additionally, severe COVID-19 patients present with persistent fever, pancytopenia and multiorgan dysfunction, known as multisystem inflammatory syndrome (MIS) [3]. Several reports have indicated a close relationship between MIS and HLH [4, 5]. Unfortunately, we could not obtain detailed information of causes of HLH before and during the COVID-19 pandemic, such as infection (including COVID-19), malignancy, or autoimmune disease. Second, we could not perform sufficient statistical analysis because we only analyzed annual numbers of patients with each disease over a five-year period (2016-2020). Finally, the observation period was too short. Since vaccination against COVID-19 for those over 12 years of age began in February 2021 in Japan, we could not analyze the effect of vaccination on the incidence of HLH, IM, and aITP. Further studies are required to clarify the association between the incidence of HLH and the COVID-19 pandemic.

Supplementary Information The online version contains supplementary material available at https://doi.org/10.1007/s12185-022-03302-2.

Acknowledgements The authors would like to thank all HLH/LCH committee members of the Japan Children's Cancer Group (JCCG), the Histiocytosis Committee of JSPHO, and the Academic and Statistical Research Committee of JSH. 
Funding This study was supported by a Grant for Practical Research for Innovative Cancer Control from the Japan Agency for Medical Research and Development (AMED) (21ck0106717h0001).

\section{Declarations}

Conflict of interest The authors declare that they have no conflict of interest.

\section{References}

1. Ishii E, Ohga S, Imashuku S, Yasukawa M, Tsuda H, Miura I, et al. Nationwide survey of hemophagocytic lymphohistiocytosis in Japan. Int J Hematol. 2007;86:58-65.

2. Chen N, Zhou M, Dong X, Qu J, Gong F, Han Y, et al. Epidemiological and clinical characteristics of 99 cases of 2019 novel coronavirus pneumonia in Wuhan, China: a descriptive study. The Lancet. 2020;395:507-13.

3. Rowley AH. Understanding SARS-CoV-2-related multisystem inflammatory syndrome in children. Nat Rev Immunol. 2020;20:453-4.

4. Panaro S, Cattalini M. The Spectrum of Manifestations of Severe Acute Respiratory Syndrome-Coronavirus 2 (SARS-CoV2) Infection in Children: What We Can Learn From Multisystem Inflammatory Syndrome in Children (MIS-C). Front Med (Lausanne). 2021;8:747190.

5. Retamozo S, Brito-Zerón P, Sisó-Almirall A, Flores-Chávez A, Soto-Cárdenas M-J, Ramos-Casals M. Haemophagocytic syndrome and COVID-19. Clin Rheumatol. 2021;40:1233-44.

Publisher's Note Springer Nature remains neutral with regard to jurisdictional claims in published maps and institutional affiliations. 